\title{
Erratum to: Robotic Urology
}

\author{
Marco Randazzo, Christian Padevit, \\ and Hubert John
}

\section{Erratum to:}

Chapter 46 in: H. John, P. Wiklund (eds.), Robotic Urology, https://doi.org/10.1007/978-3-319-65864-3_46

In this book chapter 46 was replaced with the updated version. Also, FM and Index was updated. The original book has been updated accordingly. 